\title{
Numerical magnitude processing in abacus-trained children with superior mathematical ability: an EEG study*
}

\author{
Jian HUANG ${ }^{\S 1}$, Feng-lei DU ${ }^{\S 1}$, Yuan YAO $^{1,2}$, Qun WAN ${ }^{1,2}$, Xiao-song WANG ${ }^{3}$, Fei-yan CHEN $^{\dagger \neq 1}$ \\ ( ${ }^{1}$ Bio-X Laboratory, Department of Physics, Zhejiang University, Hangzhou 310027, China) \\ ( ${ }^{2}$ Department of Psychology and Behavioral Sciences, Zhejiang University, Hangzhou 310028, China) \\ $\left({ }^{3}\right.$ Heilongjiang Abacus Association, Haerbin 150001, China) \\ †E-mail: chenfy@zju.edu.cn \\ Received Oct. 27, 2014; Revision accepted June 8, 2015; Crosschecked July 8, 2015
}

\begin{abstract}
Distance effect has been regarded as the best established marker of basic numerical magnitude processes and is related to individual mathematical abilities. A larger behavioral distance effect is suggested to be concomitant with lower mathematical achievement in children. However, the relationship between distance effect and superior mathematical abilities is unclear. One could get superior mathematical abilities by acquiring the skill of abacus-based mental calculation (AMC), which can be used to solve calculation problems with exceptional speed and high accuracy. In the current study, we explore the relationship between distance effect and superior mathematical abilities by examining whether and how the AMC training modifies numerical magnitude processing. Thus, mathematical competencies were tested in 18 abacus-trained children (who accepted the AMC training) and 18 non-trained children. Electroencephalography (EEG) waveforms were recorded when these children executed numerical comparison tasks in both Arabic digit and dot array forms. We found that: (a) the abacus-trained group had superior mathematical abilities than their peers; (b) distance effects were found both in behavioral results and on EEG waveforms; (c) the distance effect size of the average amplitude on the late negative-going component was different between groups in the digit task, with a larger effect size for abacus-trained children; (d) both the behavioral and EEG distance effects were modulated by the notation. These results revealed that the neural substrates of magnitude processing were modified by AMC training, and suggested that the mechanism of the representation of numerical magnitude for children with superior mathematical abilities was different from their peers. In addition, the results provide evidence for a view of non-abstract numerical representation.
\end{abstract}

Key words: Electroencephalography (EEG), Abacus training, Distance effect, Numerical magnitude processing, Child doi: $10.1631 /$ jzus.B1400287 Document code: A CLC number: B842

\section{Introduction}

The processing of numerical magnitude is crucial for individual mathematical ability (Butterworth, 2005 ) and is thought to be a key precursor of math-

\footnotetext{
* Corresponding author

${ }^{\S}$ The two authors contributed equally to this work

* Project supported by the National High-Tech R\&D Program (863) of China (Nos. 2012AA011603 and 2012AA011602) and the National Natural Science Foundation of China (Nos. 30900389 and 31270026 ) (10RCID: Jian HUANG, http://orcid.org/0000-0002-7259-3066

C Zhejiang University and Springer-Verlag Berlin Heidelberg 2015
}

ematical competencies (Ansari and Karmiloff-Smith, 2002). One of the best established markers of numerical magnitude processing is the distance effect (DE) (Szũcs et al., 2007; Soltész et al., 2011): the larger the distance between two numbers, the easier it is to discriminate between them. This effect was first reported by Moyer and Landauer (1967), and then replicated in adults (Henik and Tzelgov, 1982; Dehaene et al., 1990; Turconi et al., 2004), children (Szũcs et al., 2007; Soltész et al., 2011), and individuals with mathematical disabilities (Price et al., 2007; Mussolin et al., 2010a; 2010b; Furman and Rubinsten, 2012; Heine et al., 2013). This means that 
DEs exist commonly and across all ages in numerical magnitude processes. Neuroimaging studies suggest that the horizontal intraparietal sulcus (HIPS) was found to be modulated by the numerical difference between numerals in the human brain (Dehaene et al., 2004; Ansari et al., 2005; Mussolin et al., 2010a). Electroencephalography (EEG) studies also detected DEs in adults (Dehaene, 1996), children (Temple and Posner, 1998; Szũcs et al., 2007), and adolescents with developmental dyscalculia (Szũcs et al., 2007), and revealed that voltage differences on late components in occipito-parietal regions (on occipito-parietal electrodes) were associated with numerical distance in numerical comparison tasks (Dehaene, 1996; Temple and Posner, 1998; Szũcs et al., 2007).

Researches focused on the relationship between DEs and individual numerical abilities reported that larger behavioral DEs were thought to be concomitant with lower mathematical achievement in children (de Smedt et al., 2009; Holloway and Ansari, 2009). This relationship was also evidenced in studies on children with mathematical disabilities or developmental dyscalculia, which showed that children with mathematical disabilities have particular deficits in the understanding and processing of numerical magnitudes (Landerl et al., 2004). Imaging studies also found an age-related increase of distance-related activation in the inferior parietal cortex for numerical magnitude comparisons (Ansari et al., 2005). EEG studies focused on the DEs showed that differences between adolescents with developmental dyscalculia and the control were located on parietal electrodes in the late component of the EEG waveforms (400 and $440 \mathrm{~ms}$ ) (Soltész et al., 2007). All these studies indicated that the DEs of children with superior mathematical achievement should be different from the normal. However, this has not been confirmed. In the current study, we adopted the numerical magnitude comparison task and investigated the differences between the DEs of typically developed children and a special group of children who acquired superior mathematical abilities through abacus-based mental calculation (AMC) training.

$\mathrm{AMC}$ is a unique strategy for arithmetic, which can be used to solve calculation problems with exceptional speed and high accuracy (Hatano et al., 1977). AMC experts acquire their capabilities through long-term training. Initially, they learn to perform calculations on an abacus (a simple device consisting of beads and rods, and where numbers can be represented by the spatial locations of beads) with both hands simultaneously. The schematic of an abacus and the procedure of solving a calculation problem are shown in Fig. 1a. After becoming familiar with the operation of the abacus, they are instructed to imagine moving beads with actual finger movements on an imagined abacus while watching a real one. Finally, they calculate via an imagined abacus without moving fingers, as if manipulating a "mental abacus".

Previous studies, which explored the neural mechanisms of AMC, have found that the "mental abacus" was represented in the visuospatial regions located in parietal regions of the brain (Hanakawa et al., 2003; Chen et al., 2006; Frank and Barner, 2012; Du et al., 2013). Moreover, Frank and Barner (2012) revealed that the "mental abacus" was represented by a column-based model, in which the abacus is split into a series of columns and each column is independently stored as a unit with its own detailed substructure. Our previous study implied that this special visuospatial representation of numerals may result in greater numerical processing efficiency for experienced abacus-trained children than for their peers (Wang et al., 2013).

However, the DE that reflects basic numerical magnitude processing was rarely studied for AMC trainees, especially in the time course. Based on results of previous studies that focused on basic numerical magnitude processes and AMC experts, we assumed that the DE of abacus-trained children should be different from that of their peers. Therefore, by applying EEG with its high temporal resolution, we explored the relationship between DE and superior mathematical abilities by examining whether and how the AMC training modifies the numerical magnitude processing. According to the DE-related EEG results mentioned above (Dehaene, 1996; Temple and Posner, 1998; Soltész et al., 2007), we assumed that the EEG differences between the DEs of abacustrained children and their peers may be located on occipito-parietal electrodes (Dehaene, 1996; Temple and Posner, 1998) and probably on late components (Soltész et al., 2007). 


\section{Materials and methods}

\subsection{Subjects}

Two groups of children participated in the study, consisting of an abacus-trained group ( 8 boys and 10 girls, mean age (7.96 \pm 0.40$)$ years, range $7.30-8.61$ years) and an age- and education-matched non-trained group ( 9 boys and 9 girls, mean age $(7.93 \pm 0.54$ ) years, range $7.03-8.77$ years). These children were recruited from different classes of the same grade in the same school. At the beginning of their schooling, all children were randomly assigned into two experimental groups. The abacus-trained group received AMC training for about $2 \mathrm{~h}$ per week for approximately one and a half years in special mathematics classes at school. The non-trained group received normal mathematics classes at school and received no AMC training either at school or after school.

All participants were urban right-handed Chinese children with normal or corrected-to-normal vision, and reported no history of neurological or psychiatric disorders. This study was approved by Zhejiang University (China). Written informed consent was obtained from the guardian of each subject.

\subsection{Behavioral tests}

The basic mathematical competencies of children were assessed with the Chinese Rating Scale of Basic Mathematical Competencies at the Elementary School Level (Wu and Li, 2006), which was modified from the Heidelberg Mathematics Test (Haffner et al., 2005). Children's intelligence quotients (IQs) were also measured using the Chinese version of the Raven's Standardized Reasoning Test (Zhang and Wang, 1985).

\subsection{Stimuli and tasks}

To begin each trial, subjects fixated on the center of a 17-inch computer monitor. Stimuli consist of either one of the digits, $2,4,6$, and 8 or arrays of dots corresponding to those digits. Digits were presented with a font-size of 40 ; arrays of dots were randomly assigned into a $3 \times 3$ grid (the lines of the grid were hidden). Numbers were presented in a random order. The visual angle of numbers was about $4^{\circ} \times 4^{\circ}$. Stimuli were presented using E-Prime (Version 1.2) with a display mode of $800 \times 600$ pixels. Each trial started with a fixation cross $(500 \mathrm{~ms})$, followed by a number, and ended with another fixation cross $(500 \mathrm{~ms})$. Subjects were asked to press a key as rapidly as pos- sible to indicate whether the stimulus was larger or smaller than 5 . The assignment of larger or smaller to keys was balanced over subjects. Stimuli remained on the screen until the button was pressed. The test consisted of a practice set of 20 trials and 4 blocks of 60 recorded trials per subject.

\subsection{EEG data acquisition}

The EEG was recorded from 64 sites according to the international 10-20 system using a Neuroscan Synamps2 amplifier and data acquisition software. The vertical electrooculogram (EOG) was recorded from electrodes attached above and below the left eye, and the horizontal EOG from the outer canthi of both eyes. The reference electrode was placed at the left mastoid. The ground electrode was placed at a point midway between Fpz and Fz electrodes. Skin impedances were kept below $5 \mathrm{k} \Omega$. The EEG was sampled at $1000 \mathrm{~Hz}$, band-pass filtered from 0.01 to $100 \mathrm{~Hz}$, and notch-filtered for $50 \mathrm{~Hz}$. During the data recording, subjects were told to fixate on the center of the monitor, keep their bodies steady, and minimize movement of eyes and facial muscles.

\subsection{Statistical analysis}

Four children's (two abacus-trained children) mathematical competency scores and two children's (one abacus-trained child) IQ scores were lacking as these children were absent when the mathematical competencies and IQs were tested. Six abacus-trained children ( 4 boys) and 2 non-trained children ( 2 boys) were excluded in the EEG data analysis due to recording problems, but no behavioral data of the tasks were excluded. All statistical analyses were performed with SPSS for Windows (Version 16.0, SPSS Inc., Chicago, IL, USA).

\subsubsection{Behavioral analysis}

Scores of the mathematical competencies and IQs of the two groups were subjected to independent sample Student's $t$-tests to determine group differences separately. The median reaction time (RT) was calculated for each participant in each condition on correct trials only. These correct median RTs (also accuracies) were subjected as a dependent variable to repeated measures analysis of variances (ANOVAs) with notation (digit vs. dot) and distance (close to 5 vs. far from 5) as within-subjects factors and group as a between-subjects factor. 


\subsubsection{EEG analysis}

EEG and EOG data were processed with the Scan 4.3.3 software (Neuroscan Inc., El Paso, TX, USA). EEG waveforms were corrected for EOG interference and re-referenced to the average of all electrodes. Epochs were extracted from -200 to $800 \mathrm{~ms}$ relative to the stimulus onset and baseline-corrected relative to the -200 to $0 \mathrm{~ms}$ interval. Trials with EEG activity exceeding $\pm 120 \mu \mathrm{V}$ or with an incorrect response were excluded from further analysis. Remaining trials were averaged for each type of stimulus separately for each subject. Average waveforms were filtered with a low-pass filter of $30 \mathrm{~Hz}$.

Based on visual inspection of grand average waveforms and previous EEG studies on numerical processing (Dehaene, 1996; Temple and Posner, 1998; Heine et al., 2013), we selected the N1 component, the P300 component, and the late negative-going component (interval: $350-650 \mathrm{~ms}$ ) on occipito-parietal electrodes (PO7 and PO8) for the statistical analysis. Peak latencies and amplitudes for N1 and P300 and the mean amplitudes of the late negative-going component were subjected to repeated measures ANOVAs with notation (digit vs. dot) and distance (close vs. far) as within-subjects factors and group as a between-subjects factor for both PO7 and PO8 electrodes.

\section{Results}

\subsection{Behavior data}

The analysis of mathematical competency scores revealed a significant difference between the two groups with a larger value for the abacus-trained group ( $t=5.045, p<0.001$, Cohen's $d=1.559 ; T$ scores were $58.54 \pm 6.05$ and $47.15 \pm 6.70$ for the abacustrained and non-trained children, respectively; Cohen's $d$ is an effect size used to indicate the difference between two means), while no difference was found between groups in the IQ analysis $(t=1.226, p=0.229$, Cohen's $d=0.420$ ).

The analysis of RT revealed significant effects of notation $\left(F(1,34)=19.88, p<0.001, \eta_{p}{ }^{2}=0.369 ; F\right.$ is a value resulting from a statistical test used in ANOVA, and $\eta_{p}{ }^{2}$ represents the "partial eta-squared", which describes the percentage of variance explained in the dependent variable by a predictor while con- trolling for other predictors $)$ and distance $(F(1,34)=$ $\left.112.85, p<0.001, \eta_{p}{ }^{2}=0.768\right)$. Of the interactions, only notation by distance was significant $(F(1,34)=46.09$, $\left.p<0.001, \eta_{p}{ }^{2}=0.575\right)$. Decomposition into contrasts showed that DEs were all significant in both notations (digit: $F(1,34)=24.21, p<0.001, \eta_{p}{ }^{2}=0.416$; dot: $\left.F(1,34)=111.79, p<0.001, \eta_{p}{ }^{2}=0.767\right)$, with larger RT in the close condition than in the far condition. The DEs in each notation (digit vs. dot) in both groups are shown in Fig. 1b.

The analysis of accuracies revealed that only the DE was significant $\left(F(1,34)=9.10, p=0.005, \eta_{p}{ }^{2}=\right.$ $0.211)$. No interactions reached significance $(p \mathrm{~s}>0.05)$.

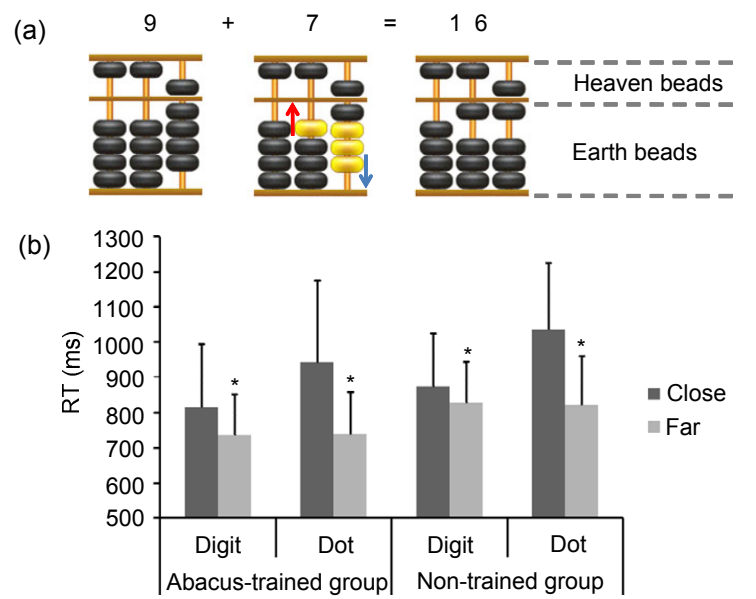

Fig. 1 Abacus addition procedure and behavioral results of distance effect (DE) in all conditions

(a) An addition example on the abacus $(9+7=16)$. The left abacus schematic represents the number 9 (one heaven bead equals to 5 and 4 earth beads equal to 4 ). The middle abacus schematic represents the addition procedure: subtract the complement of the addend to 10 ( 3 here) by pushing down the 3 yellow beads (near the blue arrow) with the index finger, then add 1 to the tens column by pushing up the yellow bead (near the red arrow) with the thumb. The right abacus schematic represents the result. (b) Behavioral results show decreasing reaction time (RT) for increasing numerical distance in two notations (Arabic digits and dot digits) in both groups. Data are expressed as mean (standard deviation), with $n=18$. ${ }^{*} p<0.001$, vs. close (Note: for interpretation of the references to color in this figure legend, the reader is referred to the web version of this article)

\subsection{EEG data}

For the N1 and P300 components, peak latency and amplitude (Tables 1 and 2) analyses were undertaken. For the late negative-going component, the average amplitude (interval: $350-650 \mathrm{~ms}$ ) analysis was made. The average amplitudes for each condition are shown in Table 3. 
Table 1 Peak latency of the N1 and P300 for all conditions

\begin{tabular}{|c|c|c|c|c|c|}
\hline \multirow{3}{*}{ Group } & \multirow{3}{*}{ Electrode } & \multicolumn{4}{|c|}{ Peak latency ${ }^{*}(\mathrm{~ms})$} \\
\hline & & \multicolumn{2}{|c|}{ Arabic digit } & \multicolumn{2}{|c|}{ Dot digit } \\
\hline & & Close & Far & Close & Far \\
\hline \multirow{6}{*}{$\begin{array}{l}\text { Abacus-trained } \\
(n=12)\end{array}$} & N1 & & & & \\
\hline & PO7 & $229 \pm 20.40$ & $235 \pm 18.22$ & $210 \pm 16.40$ & $207 \pm 22.35$ \\
\hline & PO8 & $219 \pm 18.99$ & $222 \pm 17.84$ & $204 \pm 11.74$ & $211 \pm 17.00$ \\
\hline & P300 & & & & \\
\hline & PO7 & $348 \pm 18.46$ & $343 \pm 16.32$ & $321 \pm 14.44$ & $329 \pm 27.94$ \\
\hline & PO8 & $346 \pm 19.97$ & $345 \pm 18.28$ & $327 \pm 22.44$ & $331 \pm 19.66$ \\
\hline \multirow{6}{*}{$\begin{array}{l}\text { Non-trained } \\
(n=16)\end{array}$} & N1 & & & & \\
\hline & PO7 & $232 \pm 22.04$ & $232 \pm 20.78$ & $203 \pm 15.92$ & $210 \pm 20.53$ \\
\hline & PO8 & $228 \pm 21.44$ & $229 \pm 19.83$ & $207 \pm 22.82$ & $210 \pm 19.29$ \\
\hline & P300 & & & & \\
\hline & PO7 & $356 \pm 29.69$ & $341 \pm 28.13$ & $335 \pm 29.06$ & $335 \pm 29.47$ \\
\hline & PO8 & $363 \pm 35.99$ & $347 \pm 28.57$ & $348 \pm 39.32$ & $342 \pm 30.01$ \\
\hline
\end{tabular}

${ }^{*}$ Values are expressed as mean \pm standard deviation

Table 2 Peak amplitude of the N1 and P300 for all conditions

\begin{tabular}{|c|c|c|c|c|c|}
\hline \multirow{3}{*}{ Group } & \multirow{3}{*}{ Electrode } & \multicolumn{4}{|c|}{ Peak amplitude ${ }^{*}(\mu \mathrm{V})$} \\
\hline & & \multicolumn{2}{|c|}{ Arabic digit } & \multicolumn{2}{|c|}{ Dot digit } \\
\hline & & Close & Far & Close & Far \\
\hline \multirow{6}{*}{$\begin{array}{l}\text { Abacus-trained } \\
(n=12)\end{array}$} & N1 & & & & \\
\hline & PO7 & $-8.34 \pm 3.16$ & $-9.25 \pm 4.33$ & $-6.10 \pm 5.57$ & $-5.51 \pm 4.54$ \\
\hline & PO8 & $-10.23 \pm 4.67$ & $-10.48 \pm 6.18$ & $-9.49 \pm 5.35$ & $-8.43 \pm 5.54$ \\
\hline & P300 & & & & \\
\hline & PO7 & $4.06 \pm 3.27$ & $5.42 \pm 3.79$ & $7.30 \pm 3.24$ & $7.18 \pm 2.76$ \\
\hline & PO8 & $4.25 \pm 4.70$ & $6.34 \pm 5.49$ & $6.98 \pm 4.11$ & $6.40 \pm 4.14$ \\
\hline \multirow{6}{*}{$\begin{array}{l}\text { Non-trained } \\
(n=16)\end{array}$} & $\mathrm{N} 1$ & & & & \\
\hline & PO7 & $-7.20 \pm 5.14$ & $-8.21 \pm 5.90$ & $-6.28 \pm 5.30$ & $-5.56 \pm 5.52$ \\
\hline & PO8 & $-8.78 \pm 5.68$ & $-10.00 \pm 6.94$ & $-6.97 \pm 6.77$ & $-6.65 \pm 7.05$ \\
\hline & P300 & & & & \\
\hline & PO7 & $4.11 \pm 3.87$ & $4.87 \pm 4.50$ & $5.42 \pm 4.64$ & $4.57 \pm 4.05$ \\
\hline & PO8 & $4.91 \pm 4.77$ & $4.74 \pm 5.40$ & $5.98 \pm 8.40$ & $5.60 \pm 7.00$ \\
\hline
\end{tabular}

${ }^{*}$ Values are expressed as mean \pm standard deviation

Table 3 Mean amplitude of the late negative-going component for all conditions

\begin{tabular}{lcccccc}
\hline \multirow{2}{*}{ Group } & \multirow{2}{*}{ Electrode } & \multicolumn{3}{c}{ Amplitude $^{*}(\mu \mathrm{V})$} \\
\cline { 3 - 4 } \cline { 3 - 4 } \cline { 5 - 6 } & & \multicolumn{2}{c}{ Close } & Far & & \multicolumn{2}{c}{ Close } & Fot digit \\
\hline Abacus-trained & PO7 & $-3.16 \pm 2.98$ & $-0.65 \pm 3.28$ & & $-2.05 \pm 2.93$ & $-1.77 \pm 3.52$ \\
$(n=12)$ & PO8 & $-3.43 \pm 3.51$ & $-1.91 \pm 3.01$ & & $-2.10 \pm 3.66$ & $-2.26 \pm 4.41$ \\
Non-trained & PO7 & $-0.44 \pm 2.41$ & $-0.23 \pm 1.75$ & & $-0.75 \pm 2.76$ & $-0.22 \pm 1.95$ \\
$(n=16)$ & PO8 & $-0.55 \pm 2.15$ & $-0.60 \pm 2.13$ & & $-0.36 \pm 4.18$ & $1.01 \pm 3.71$ \\
\hline
\end{tabular}

${ }^{*}$ Values are expressed as mean \pm standard deviation

\subsubsection{N1 component}

For the latency of the N1 component, the main effect of notation was significant on both $\mathrm{PO} 7$ and PO8 electrodes (PO7: $F(1,26)=49.12, p<0.001, \eta_{p}{ }^{2}=$ 0.654; PO8: $F(1,26)=28.92, p<0.001, \eta_{p}{ }^{2}=0.527$ ), with longer latencies for digit notations than for dot notations.
For the amplitude of the N1 component, significant effects of notation on both $\mathrm{PO} 7$ and $\mathrm{PO} 8$ electrodes were detected (PO7: $F(1,26)=12.33, p=0.002$, $\eta_{p}{ }^{2}=0.322$; PO8: $F(1,26)=7.29, p=0.012, \eta_{p}{ }^{2}=0.219$ ), with more negative amplitudes for digit notations than for dot notations. The two-way interaction of notation $\times$ distance was significant $(\mathrm{PO} 7: F(1,26)=$ 
6.09, $p=0.020, \eta_{p}{ }^{2}=0.190 ;$ PO8: $F(1,26)=6.39, p=$ $\left.0.018, \eta_{p}{ }^{2}=0.197\right)$. Decomposition into contrasts showed that none of the DEs was significant ( $p=$ 0.057 for the digit task and $p=0.206$ for the dot task on PO7; $p=0.255$ for the digit task and $p=0.112$ for the dot task on PO8).

\subsubsection{P300 component}

For the latency of the P300 component, the main effect of notation was significant on both PO7 and PO8 electrodes (PO7: $F(1,26)=18.04, p<0.001, \eta_{p}{ }^{2}=0.410$; PO8: $\left.F(1,26)=9.22, p=0.005, \eta_{p}{ }^{2}=0.262\right)$, with longer latencies for digit notations than for dot notations. The two-way interaction of notation $\times$ distance was significant on PO7 $\left(F(1,26)=4.83, p=0.037, \eta_{p}{ }^{2}=0.157\right)$. Decomposition into contrasts showed that the DE was statistically significant only in the digit task on PO7 $\left(F(1,26)=8.59, p=0.007, \eta_{p}{ }^{2}=0.248\right)$, with longer latencies for close conditions than for far conditions.

$\mathrm{PO}$

(a)

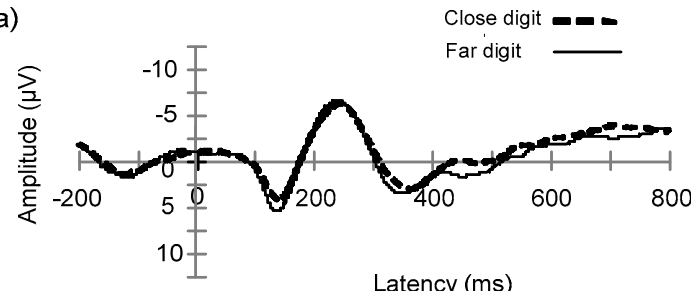

(b)

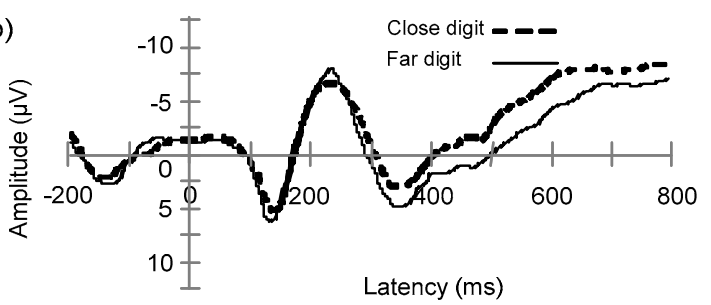

(c)

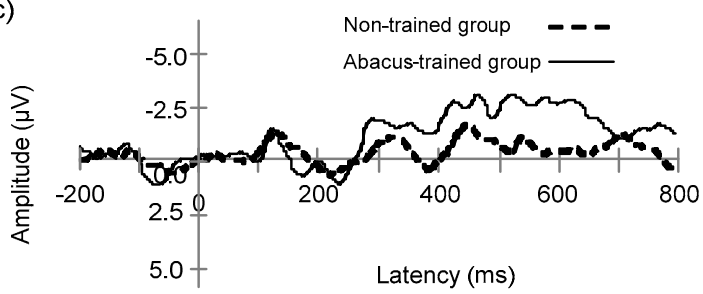

For the amplitude of the P300 component, the main effect of notation was significant on the PO7 electrode $\left(F(1,26)=10.76, p=0.003, \eta_{p}{ }^{2}=0.293\right)$, with more positive amplitudes for dot notations than for digit notations. The two-way interaction of notation $\times$ group was significant on the PO7 electrode $(F(1,26)=$ 4.74, $\left.p=0.039, \eta_{p}{ }^{2}=0.154\right)$. The two-way interaction of notation $\times$ distance was significant on the PO7 electrode $\left(F(1,26)=4.72, p=0.039, \eta_{p}{ }^{2}=0.154\right)$. Decomposition into contrasts showed that no group effects were found in both notations ( $p=0.862$ and $p=$ 0.120 for the digit and dot tasks, respectively); the DE was significant on the $\mathrm{PO} 7$ electrode in the digit task $\left(F(1,26)=4.83, p=0.037, \eta_{p}{ }^{2}=0.157\right)$, with more positive amplitudes for far conditions than for close conditions.

Grand average waveforms in the digit task were shown in Figs. $2 \mathrm{a}$ and $2 \mathrm{~b}$ for the non-trained and abacus-trained groups, respectively.
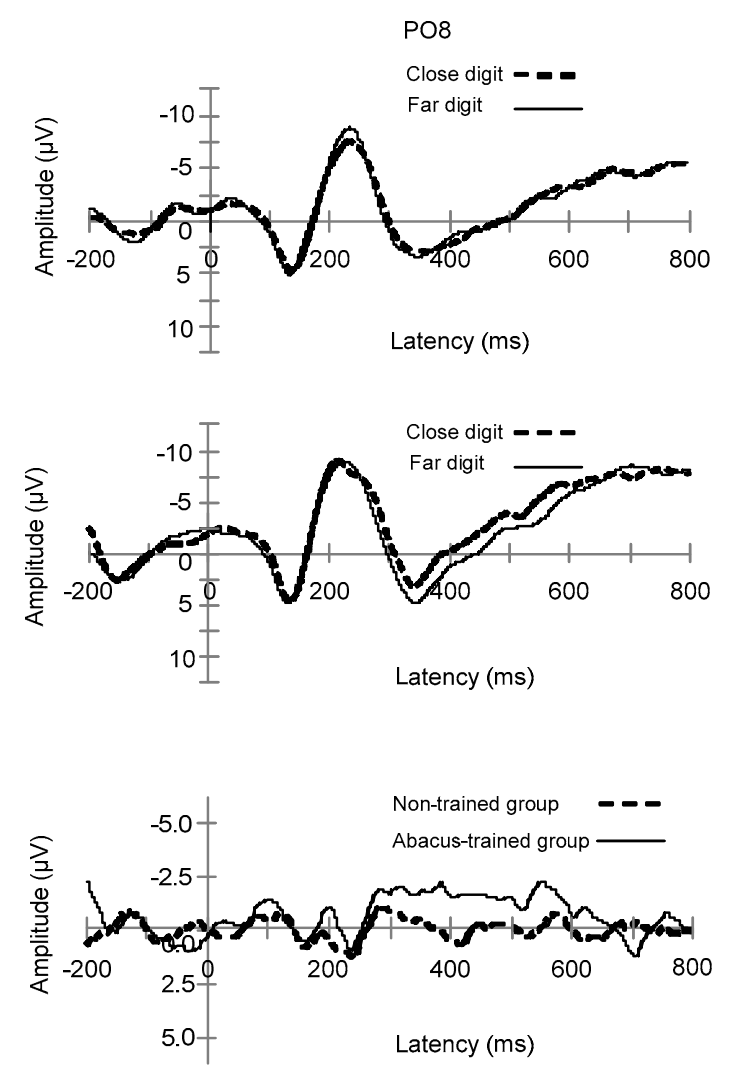

Fig. 2 Grand average and subtraction waveforms for the non-trained and abacus-trained groups in the digit task (a) Grand average waveforms of close distance and far distance for the non-trained group in the digit task on PO7 and PO8; (b) Grand average waveforms of close distance and far distance for the abacus-trained group in the digit task on PO7 and PO8; (c) Subtraction waveforms of the abacus-trained and non-trained groups in the digit task on PO7 and PO8 


\subsubsection{Late negative-going component}

For the average latency of the negative-going component (interval: $350-650 \mathrm{~ms}$ ), the main effect of distance was significant on both PO7 and PO8 electrodes (PO7: $F(1,26)=15.54, p=0.001, \eta_{p}{ }^{2}=0.374$; PO8: $\left.F(1,26)=4.92, p=0.035, \eta_{p}{ }^{2}=0.159\right)$. The main effect of group was marginally significant on PO7 $\left(F(1,26)=3.09, p=0.09, \eta_{p}{ }^{2}=0.106\right)$ and significant on PO8 $\left(F(1,26)=4.85, p=0.037, \eta_{p}{ }^{2}=0.157\right)$. The twoway interaction of notation $\times$ distance was significant on the PO7 electrode $(F(1,26)=9.17, p=0.006$, $\left.\eta_{p}{ }^{2}=0.261\right)$. The three-way interaction of notation $\times$ distance $\times$ group was significant on PO7 $(F(1,26)=$ $\left.7.14, p=0.013, \eta_{p}{ }^{2}=0.215\right)$. Further analysis showed that the distance $\times$ group interaction was significant in the digit task (PO7: $F(1,26)=9.03, p=0.006, \eta_{p}{ }^{2}=$ $0.258)$. The DE was only statistically significant in the digit task on PO7 in abacus-trained group $(F(1,11)=$ $\left.23.88, p<0.001, \eta_{p}{ }^{2}=0.685\right)$, with more negative amplitudes for close conditions than for far conditions.

To further examine the interaction of notation $\times$ distance $\times$ group, the size of DE was calculated as the difference between the amplitudes of the close condition and the far condition. These DE sizes were then subjected to repeated measures ANOVAs with notation (digit vs. dot) as a within-subjects factor and group as a between-subjects factor. Results showed that the effect of notation was significant on the PO7 electrode $\left(F(1,26)=7.03, p=0.006, \eta_{p}{ }^{2}=0.261\right)$. The two-way interaction of notation $\times$ group was significant on the PO7 electrode $(F(1,26)=7.14, p=0.013$, $\left.\eta_{p}{ }^{2}=0.215\right)$. Decomposition into contrasts showed that the group effect was significant only in the digit on PO7 ( $t=-3.00, p=0.006$, Cohen's $d=-1.30)$, with larger DE size for the abacus-trained group than for the non-trained group. Subtraction waveforms of the abacus-trained and non-trained groups in the digit task are shown in Fig. 2c.

\section{Discussion}

The present study investigated the neural substrates of numerical processing in children with AMC training compared to their peers. We observed that: (a) the abacus-trained group had superior mathematical abilities than their peers; (b) DEs were found in both behavioral results and on EEG waveforms; (c) the
DE size on the amplitude of the late negative-going component was different between groups in the digit task with larger effect size for abacus-trained children, although no behavioral differences were detected between the DE sizes of the two groups in tasks of both notations; (d) both the behavioral and EEG DEs were modulated by the notation.

The mathematical competency scores of the two groups were significantly different with higher scores for abacus-trained children, indicating that the abacustrained group had superior mathematical abilities than their peers. Meanwhile, no differences were detected between the IQs of the two groups, indicating that both groups had comparative intelligence. This supported the point of view that the observed differences between groups were likely to correspond to different developmental trajectories.

In the behavioral result, both groups of children showed DEs in tasks of both notations. This result is in line with previous findings showing that items far from the standard are processed faster than the close, indicating that children can access the mental representation of the number line in a comparison task (Temple and Posner, 1998; Szũcs et al., 2007; Soltész et al., 2011). However, the interaction between group and distance was not significant, indicating that the behavioral DEs were similar for the two groups. This is dissociated from the view that larger behavioral DEs were thought to be concomitant with lower mathematical achievement in children (de Smedt et al., 2009; Holloway and Ansari, 2009). The reason for this dissociation might be that the intensity of the AMC training was not sufficient to change the behavioral DE when the numerical comparison problems were carried out. Also we should note that the sample size of the present study (36 children) is not huge according to the standards of behavioral studies, and a larger and/or more varied sample might possibly provide somewhat different results, at least in detail. This may also result in the disassociation between the present behavioral result and previous results.

Consistent with the EEG result reported by Dehaene (1996), the N1 component showed no DE. However, notation effects on the N1 latency and amplitude were considerable, supporting the idea that N1 effects were related to differences in sensory processing (Libertus et al., 2007). Additionally, similar to previous results (Grune et al., 1993; Dehaene, 1996; 
Schwarz and Heinze, 1998; Jiang et al., 2010), our study showed DEs on the occipital-parietal P300 component in the digit task, which was characterized with more positive amplitudes and shorter latencies for far conditions than for close conditions. As close conditions are harder and need more effort than far conditions, our results revealed that larger amplitudes and shorter latencies of $\mathrm{P} 300$ were corresponding to easier comparison problems (with far distance). These results confirm the view that the P300 amplitude is negatively correlated while P300 latency is positively correlated with difficulty (Picton, 1992; Paulsen and Neville, 2008).

Most importantly, DEs were statistically significant in the analysis of the mean amplitude of the late negative-going component in the digit task. Sizes of DEs were different between the two groups of children, with a larger effect size for abacus-trained children. These results support our hypothesis: the DE of abacus-trained children is different from their peers The finding suggests that the neural substrates under the magnitude processing have been modified during the course of AMC training. This is consistent with the previous conclusion of our study, which showed that substantial gains in numerical processing efficiency could be achieved through long-term intensive AMC training (Wang et al., 2013). Some earlier research suggests that abacus training need not always lead to transfer to other mathematical tasks (Hatano, 1988). This is different from the present result and the conclusion of Wang et al. (2013). The reason for this is complicated. In particular, different forms of training played an important role and may result in diverse outcomes.

However, no DEs were found in the dot task on EEG results, although behavioral results showed significant DEs. This finding supports the idea that similar behavioral effects can be produced by different mechanisms (Rumelhart et al., 1986; Cohen Kadosh et al., 2007).

Interactions between notation and distance were detected in both behavioral and EEG results, revealing that the $\mathrm{DE}$ was modulated by the notation (i.e. notation-dependent). This result challenges the commonly held view of abstract numerical representation, which suggests that the same representation exists for different notations of numbers (Mccloskey, 1992; Dehaene and Akhavein, 1995; Dehaene et al., 1998;
Schwarz and Ischebeck, 2000; Naccache and Dehaene, 2001; Pinel et al., 2001), and supports the view of non-abstract numerical representation held by Cohen Kadosh and Walsh (2009). The view of the non-abstract numerical representation is also evidenced by many studies. For example, Ganor-Stern and Tzelgov (2008) found that when subjects were asked to compare pairs of numbers for their numerical value, the DE was modulated as a function of notation. Holloway and Ansari (2009) found that the DE was only correlated with mathematical achievement when the numerical notation was in digit form (comparing to numbers appearing as squares). Together with all these studies, our findings provide evidence directly challenging the idea that numbers are represented abstractly and suggest that different developmental trajectories underlie the representation of symbolic and non-symbolic numerical magnitudes.

Overall, although no differences between the behavioral DES of the two groups were detected, the EEG waveforms did show a significant difference between the DE sizes of the two groups on the amplitude of the late negative-going component in the digit task. Together with the result that abacus-trained children have superior mathematical competencies, the present study suggests that the neural mechanism of the representation of numerical magnitude for children with superior mathematical ability may be different from that for their peers.

In conclusion, the present study showed differences in DEs on EEG data between the abacus-trained and non-trained groups in the digit task. These differences indicated that the neural substrates of magnitude processing were modified during the course of AMC training, although no differences were detected on behavioral data between groups. These results suggest that the neural mechanism of the representation of numerical magnitude for children with superior mathematical ability may be different from that for their peers. Meanwhile, our results provide powerful evidence for the view of non-abstract numerical representation, suggesting that developmental trajectories underlying the representation of symbolic and non-symbolic numerical magnitudes are not matched (Cohen Kadosh and Walsh, 2009).

However, we need to mention that there are limitations in the present study. Firstly, the sample size of the present research is not huge for behavioral 
studies. The condition of a larger sample size should be explored in the future to verify the present result. Secondly, we need to note that training forms were crucial to the outcomes of the training. Differences between the training courses in different studies may cause subtly different effects; and differences between abacus training and some other forms of training may also have somewhat different effects on brain and/or behavioral performances. This should be considered when comparing the present findings to previous results and in future training studies. Thirdly, the present study mainly focused on the field of numerical magnitude processing; the other factors, which were also considered as markers of mathematical competence such as dot enumeration (Reeve et al., 2012), should also be investigated in future studies.

\section{Acknowledgements}

Thanks to the Chinese Abacus and Mental Arithmetic Association, the Finance Departments and Abacus and Mental Arithmetic Association of Heilongjiang (China) for their kind support. Thanks also to the Jiang'an Primary School of Qiqihaer (China) for data collection.

\section{Compliance with ethics guidelines}

Jian HUANG, Feng-lei DU, Yuan YAO, Qun WAN, Xiao-song WANG, and Fei-yan CHEN declare that they have no conflict of interest.

All procedures followed were in accordance with the ethical standards of the responsible committee on human experimentation (institutional and national) and with the Helsinki Declaration of 1975, as revised in 2008 (5). Informed consent was obtained from all subjects for being included in the study.

\section{References}

Ansari, D., Karmiloff-Smith, A., 2002. Atypical trajectories of number development: a neuroconstructivist perspective. Trends Cogn. Sci., 6(12):511-516. [doi:10.1016/S13646613(02)02040-5]

Ansari, D., Garcia, N., Lucas, E., et al., 2005. Neural correlates of symbolic number processing in children and adults. Neuroreport, 16(16):1769-1773. [doi:10.1097/01.wnr. 0000183905.23396.f1]

Butterworth, B., 2005. The development of arithmetical abilities. J. Child Psychol. Psychiatry, 46(1):3-18. [doi:10. 1097/01.wnr.0000183905.23396.f1]

Chen, F., Hu, Z., Zhao, X., et al., 2006. Neural correlates of serial abacus mental calculation in children: a functional MRI study. Neurosci. Lett., 403(1-2):46-51. [doi:10. 1016/j.neulet.2006.04.041]
Cohen Kadosh, R., Walsh, V., 2009. Numerical representation in the parietal lobes: abstract or not abstract? Behav. Brain Sci., 32(3-4):313-328. [doi:10.1017/S0140525X 09990938]

Cohen Kadosh, R., Cohen Kadosh, K., Kaas, A., et al., 2007. Notation-dependent and -independent representations of numbers in the parietal lobes. Neuron, 53(2):307-314. [doi:10.1016/j.neuron.2006.12.025]

Dehaene, S., 1996. The organization of brain activations in number comparison: event-related potentials and the additive-factors method. J. Cogn. Neurosci., 8(1):47-68. [doi:10.1162/jocn.1996.8.1.47]

Dehaene, S., Akhavein, R., 1995. Attention, automaticity, and levels of representation in number processing. J. Exp. Psychol. Learn. Mem. Cogn., 21(2):314. [doi:10.1037/ 0278-7393.21.2.314]

Dehaene, S., Dupoux, E., Mehler, J., 1990. Is numerical comparison digital? Analogical and symbolic effects in two-digit number comparison. J. Exp. Psychol. Hum. Percept. Perform., 16(3):626. [doi:10.1037/0096-1523. 16.3. 626]

Dehaene, S., Dehaene-Lambertz, G., Cohen, L., 1998. Abstract representations of numbers in the animal and human brain. Trends Neurosci., 21(8):355-361. [doi:10.1016/S01662236(98)01263-6]

Dehaene, S., Molko, N., Cohen, L., et al., 2004. Arithmetic and the brain. Curr. Opin. Neurobiol., 14(2):218-224. [doi:10. 1016/j.conb.2004.03.008]

de Smedt, B., Verschaffel, L., Ghesquière, P., 2009. The predictive value of numerical magnitude comparison for individual differences in mathematics achievement. $J$. Exp. Child Psychol., 103(4):469-479. [doi:10.1016/j.jecp. 2009.01.010]

Du, F., Chen, F., Li, Y., et al., 2013. Abacus training modulates the neural correlates of exact and approximate calculations in Chinese children: an fMRI study. BioMed Res. Int., 2013:694075. [doi:10.1155/2013/694075]

Frank, M.C., Barner, D., 2012. Representing exact number visually using mental abacus. J. Exp. Psychol. Gen., 141(1):134-139. [doi:10.1037/a0024427]

Furman, T., Rubinsten, O., 2012. Symbolic and non symbolic numerical representation in adults with and without developmental dyscalculia. Behav. Brain Funct., 8(1):55. [doi:10.1186/1744-9081-8-55]

Ganor-Stern, D., Tzelgov, J., 2008. Across-notation automatic numerical processing. J. Exp. Psychol. Learn. Mem. Cogn., 34(2):430. [doi:10.1037/0278-7393.34.2.430]

Grune, K., Mecklinger, A., Ullsperger, P., 1993. Mental comparison: P300 component of the ERP reflects the symbolic distance effect. Neuroreport, 4(11):1272-1274. [doi:10.1097/00001756-199309000-00016]

Haffner, J., Baro, K., Langner, C., et al., 2005. HRT 1-4: Heidelberger Rechentest: Erfassung Mathematischer Basiskompetenzen im Grundschulalter. Hogrefe, Gottingen 
(in German).

Hanakawa, T., Honda, M., Okada, T., et al., 2003. Neural correlates underlying mental calculation in abacus experts: a functional magnetic resonance imaging study. Neuroimage, 19(2):296-307. [doi:10.1016/S1053-8119(03)00050-8]

Hatano, G., 1988. Social and motivational bases for mathematical understanding. NDCAD, 1988(41):55-70.

Hatano, G., Miyake, Y., Binks, M.G., 1977. Performance of expert abacus operators. Cognition, 5(1):47-55. [doi:10. 1016/0010-0277(77)90016-6]

Heine, A., Wißmann, J., Tamm, S., et al., 2013. An electrophysiological investigation of non-symbolic magnitude processing: numerical distance effects in children with and without mathematical learning disabilities. Cortex, 49(8):2162-2177. [doi:10.1016/j.cortex.2012.11.009]

Henik, A., Tzelgov, J., 1982. Is three greater than five: the relation between physical and semantic size in comparison tasks. Mem. Cognit., 10(4):389-395. [doi:10.3758/ BF03202431]

Holloway, I.D., Ansari, D., 2009. Mapping numerical magnitudes onto symbols: the numerical distance effect and individual differences in children's mathematics achievement. J. Exp. Child Psychol., 103(1):17-29. [doi:10.1016/j.jecp.2008.04.001]

Jiang, T., Qiao, S., Li, J., et al., 2010. Effects of symbol type and numerical distance on the human event-related potential. Neuropsychologia, 48(1):201-210. [doi:10. 1016/j.neuropsychologia.2009.09.005]

Landerl, K., Bevan, A., Butterworth, B., 2004. Developmental dyscalculia and basic numerical capacities: a study of 8-9-year-old students. Cognition, 93(2):99-125. [doi:10. 1016/j.cognition.2003.11.004]

Libertus, M.E., Woldorff, M.G., Brannon, E.M., 2007. Electrophysiological evidence for notation independence in numerical processing. Behav. Brain Funct., 3(1):1-15. [doi:10.1186/1744-9081-3-1]

Mccloskey, M., 1992. Cognitive mechanisms in numerical processing: evidence from acquired dyscalculia. Cognition, 44(1-2):107-157. [doi:10.1016/0010-0277(92)90052-J]

Moyer, R.S., Landauer, T.K., 1967. Time required for judgements of numerical inequality. Nature, 215(5109):1519-1520. [doi:10.1038/2151519a0]

Mussolin, C., de Volder, A., Grandin, C., et al., 2010a. Neural correlates of symbolic number comparison in developmental dyscalculia. J. Cogn. Neurosci., 22(5):860-874. [doi:10. 1162/jocn.2009.21237]

Mussolin, C., Mejias, S., Noël, M.P., 2010b. Symbolic and nonsymbolic number comparison in children with and without dyscalculia. Cognition, 115(1):10-25. [doi:10. 1016/j.cognition.2009.10.006]

Naccache, L., Dehaene, S., 2001. Unconscious semantic priming extends to novel unseen stimuli. Cognition, 80(3): 215-229. [doi:10.1016/S0010-0277(00)00139-6]

Paulsen, D.J., Neville, H.J., 2008. The processing of non- symbolic numerical magnitudes as indexed by ERPs. Neuropsychologia, 46(10):2532-2544. [doi:10.1016/j. neuropsychologia.2008.04.003]

Picton, T.W., 1992. The P300 wave of the human event-related potential. J. Clin. Neurophysiol., 9(4):456-479. [doi:10. 1097/00004691-199210000-00002]

Pinel, P., Dehaene, S., Riviere, D., et al., 2001. Modulation of parietal activation by semantic distance in a number comparison task. Neuroimage, 14(5):1013-1026. [doi:10. 1006/nimg.2001.0913]

Price, G.R., Holloway, I., Räsänen, P., et al., 2007. Impaired parietal magnitude processing in developmental dyscalculia. Curr. Biol., 17(24):R1042-R1043. [doi:10.1016/j.cub. 2007.10.013]

Reeve, R., Reynolds, F., Humberstone, J., et al., 2012. Stability and change in markers of core numerical competencies. J. Exp. Psychol. Gen., 141(4):649. [doi:10. 1037/a0027520]

Rumelhart, D.E., Mcclelland, J.L., Group, P.R., 1986. Parallel Distributed Processing: Explorations in the Microstructure of Cognition, Vol. 1: Foundations. MIT Press, Cambridge, MA, p.560-567.

Schwarz, W., Heinze, H.J., 1998. On the interaction of numerical and size information in digit comparison: a behavioral and event-related potential study. Neuropsychologia, 36(11): 1167-1179. [doi:10.1016/S0028-3932(98)00001-3]

Schwarz, W., Ischebeck, A., 2000. Sequential effects in number comparison. J. Exp. Psychol. Hum. Percept. Perform., 26(5):1606. [doi:10.1037/0096-1523.26.5.1606]

Soltész, F., Szücs, D., Dékány, J., et al., 2007. A combined event-related potential and neuropsychological investigation of developmental dyscalculia. Neurosci. Lett., 417(2): 181-186. [doi:10.1016/j.neulet.2007.02.067]

Soltész, F., Goswami, U., White, S., et al., 2011. Executive function effects and numerical development in children: behavioural and ERP evidence from a numerical stroop paradigm. Learn. Individ. Differ., 21(6):662-671. [doi:10. 1016/j.lindif.2010.10.004]

Szũcs, D., Soltész, F., Jármi, E., et al., 2007. The speed of magnitude processing and executive functions in controlled and automatic number comparison in children: an electro-encephalography study. Behav. Brain Funct., 3(1):23. [doi:10.1186/1744-9081-3-23]

Temple, E., Posner, M.I., 1998. Brain mechanisms of quantity are similar in 5-year-old children and adults. PNAS, 95(13):7836-7841. [doi:10.1073/pnas.95.13.7836]

Turconi, E., Jemel, B., Rossion, B., et al., 2004. Electrophysiological evidence for differential processing of numerical quantity and order in humans. Cogn. Brain Res., 21(1):22-38. [doi:10.1016/j.cogbrainres.2004.05.003]

Wang, Y., Geng, F., Hu, Y., et al., 2013. Numerical processing efficiency improved in experienced mental abacus children. Cognition, 127(2):149-158. [doi:10.1016/j.cognition. 2012.12.004] 
Wu, H.R., Li, L., 2006. Norm establishment for Chinese rating scale of pupil's mathematics abilities. J. Clin. Rehab. Tissue Eng. Res., 10(30):168-171 (in Chinese).

Zhang, H., Wang, X., 1985. The Chinese Version of the Raven's Standard Progressive Matrices. Beijing Nor. Univ., Beijing, China (in Chinese).

\section{中文概要}

题 目: 高数学能力珠心算儿童数量表征的脑电研究

目 的: 阐明珠心算儿童的高数学能力与数字距离效应之 间的关系。

创新点：以经过珠心算训练的儿童为研究对象, 探索通过
珠心算训练获得的高数学能力与数字距离效应 (表征数量加工的重要标志) 的关系及珠心算儿 童拥有高数学能力的神经基础。

方 法: 实验对象选取经过一年半珠心算训练的儿童, 实 验任务为数字比较任务 (数字形式分为阿拉伯数 字和圆点两种）, 在执行数字比较任务的同时记 录儿童的脑电信息, 对不同组别不同数字形式任 务的行为学和脑电数据进行对比分析。

结 论: 通过珠心算训练而获得高数学能力儿童表征数量 信息的神经基础与普通儿童之间会存在差异, 说 明珠心算训练可能改变儿童加工数量信息的神 经基础。

关键词: 脑电图; 珠算训练; 距离效应; 数量加工; 儿童 